\title{
Small Is Beautiful: Health Care With a Human Face
}

\author{
David Loxterkamp, $M D$
}

(J Am Board Fam Med 2021;34:264-265.)

Man is small, and, therefore, small is beautiful. ${ }^{1}$ -E. F. Schumacher

As I was preparing to enter family practice training in 1979, I read 2 books that left an indelible stamp on my thinking about health and health care. The first was Wendell Berry's The Unsettling of America; the second, E. F. Schumacher's Small Is Beautiful. Schumacher was a British statistician and environmentalist who saw a danger in our drive to create larger, ever more sophisticated and unregulated technology and in the distortion of the social structure that was required to sustain it. "Ever bigger machines," he observed," do not represent progress. Wisdom demands a new orientation of science and technology toward the organic, the gentle, the nonviolent, the elegant and beautiful." 1

Schumacher was not resisting technology per se, for he could see the remarkable tools it had given doctors and hospitals for diagnosing and treating disease. Instead, he was calling for appropriate, intermediate technologies- "technology with a human face"- that could bring about enormous good in small units of production that enlivened the communities that sheltered them.

The Robert Graham Center's most recent policy brief draws attention to the decreasing number of family physicians who work in solo or small ambulatory practices. Whether the trend can be reserved, or should be, is beyond the scope of their report. But no reader should be surprised. The last several decades have seen a relentless expansion in corporate ownership and consolidation in health care, loan forgiveness programs that require employment in nonprofit entities, and a paucity of training opportunities in the smaller, less-resourced rural practices.

From the Seaport Community Health Center, Belfast, ME. Corresponding author: David A. Loxterkamp, MD, MA, Seaport Community Health Center, Belfast, ME 04915 (E-mail: david.loxterkamp@gmail.com).
Yet size matters. And while its impact on quality of care can be debated, patients as a whole prefer the intimacy of a smaller practice. So do those who work there. The problem is not one of communication, nor is it amenable to a technological solution. It has to do with our human capacity to track social relationships. Ethnographic research has shown that the average size of a hunter-gatherer overnight camp was 38 , a village was 148 , and the tribe was roughly a thousand. ${ }^{2}$ As my own "camp" grew in size, I found that I could no longer remember the names or job descriptions of my fellow employees; they, too, had difficulty remembering the names of our patients. It became nearly impossible to schedule all-staff meetings, and when we did, they were not very satisfying. Turnover accelerated. The office became more of a business, less a home.

Excessive size not only distorts human relationships within a practice but demands structural changes that compound it. The front office is divided from the back; services are sent off-site; examination rooms are made uniform, windowless, and sterile. We reward clinicians with a separate office that further isolates them. And when employees are not happy being here, why should their patients be?

The disappearance of solo and small practices has had a particular impact on rural America. This should matter to us not only because rural communities are an important constituency but because they are part of our psyche. As Gayle Stephens once noted, ${ }^{3}$ agrarianism was one of the reforming principles that gave birth to the specialty. Many a family medicine residency program or department was launched on the funding authorized by rural state legislatures in the 1960s and 1970s.

The practice of family medicine-its scope and composition-has changed dramatically since 1969 . The country, too, has changed, becoming older, less rural, more educated, and increasingly dependent on the Internet and its hidden algorithms. Any redesign of our training curriculum must bend to 
both realities. The changes that our new graduates will experience over the course of their careers will be no less dramatic than what a newly minted, business-naive family doctor encountered in 1984.

When I arrived in my adopted rural community, I not only desired to practice full-spectrum family medicine but was expected to. A colleague and I would break from the solo practice tradition and form the first partnership in Waldo County. We hired our first behavioral therapist in 1991; implemented the first of 4 electronic health record platforms in 1992; moved 3 times; became a rural health center in 1996 and a Federally Qualified Health Center in 2014; did our best to meet local needs during the AIDS epidemic and opioid crisis; participated in the National Demonstration Project and became a Level III Patient-Centered Medical Home. And in 2014 we joined the sell-off of private practice to corporate hands.

Our decisions did not flow from careful study or expert opinion. We simply tried to listen to our patients, respond to their problems, and survive. Neither the supply of patients nor their problems have gone away, and a host of questions remain:

- What kind of health care services do rural Americans really need or say they need?

- How will doctors adjust to being the minority health care provider in rural areas?

- What minimal practice size meets the basic needs of both providers and patients?

- What are the obstacles to practice innovation, local control, and the creation of truly supportive practice environments?

To be sure, technology has a place in rural medicine; helicopter transports, Project Echo, and telemedicine encounters are now standard fare. Other issues are more social in nature. For example, more than 4325 applicants entered a family medicine residency program this year. ${ }^{4}$ By comparison, more than 30,000 nurse practitioners ${ }^{5}$ and 10,000 physician assistants entered the workforce, the majority of whom remain in primary care. Family doctors fully understand that we must work in teams, but what is our role there? How should the team function?

The biggest challenge facing rural America may be the inability or unwillingness of our health care administrators to understand the peculiar needs of each community. Agrarian values are not that difficult to grasp, but it helps if you live there. For a long time. On the other hand, rural people are more than happy to educate us, as I and so many of my colleagues have learned over the years. Tom Lundquist, a recently retired family physician from Rice Lake, Wisconsin, offers this advice:

Students going into rural healthcare should know that most rural residents are self-sufficient, hardworking, family-centered, proud, full of practical knowledge, and a little suspicious of know-it all types. It therefore behooves the doctor to be humble, live simply, non-ostentatiously, and above all be interested in the lives of our patients. (personal correspondence)

The insights of E. F. Schumacher apply not only to rural economies and communities. He understood that large-scale organization was here to stay and that the principal task of the administrator was to achieve smallness within the larger organization. "People," he believed, "can be themselves only in small comprehensible groups." For there is a human scale on which communication, collaboration, and trust can flourish, and the pursuit of health will always hinge on our human capacity to influence, support, and love one another.

Communities are people, too. For those who oversee their health care, let us first walk a mile in their shoes. The size of the shoe is not critical, nor is the name we stamp on it. What matters is our determination to give agency and support to each of these communities, large or small, and to create a culture of health in the practices that serve them.

To see this article online, please go to: http://jabfm.org/content/ 34/2/264.full.

\section{References}

1. Schumacher EF. Small is beautiful: a study of economics as if people mattered. New York: Harper \& Row; 1975.

2. Stephens GG. Family medicine as counterculture. Fam Med Teacher 1979;11:14-8.

3. Match results for family medicine. 2020. Available from: https://www.aafp.org/students-residents/residencyprogram-directors/national-resident-matching-programresults.html.

4. American Association of Nurse Practitioners. NP fact sheet. 2020. Available from: https://www.aanp. org/about/all-about-nps/np-fact-sheet.

5. National Commission on Certification of Physician Assistants. 2019 statistical profile of certified physician assistants. 2020. Available from: https:// prodcmsstoragesa.blob.core.windows.net/uploads/files/ 2019StatisticalProfileofCertifiedPhysicianAssistants.pdf. 